\title{
Tumor rupture of gastric gastrointestinal stromal tumors during endoscopic resection: a risk factor for peritoneal metastasis?
}

\section{(ㄷ)(1) $\odot(9$}

\author{
Authors \\ Shiyi Song ${ }^{1}$, Wei Ren ${ }^{2}$, Yi Wang ${ }^{1}$, Shu Zhang ${ }^{1}$, Song Zhang ${ }^{1}$, Fei Liu ${ }^{1}$, Qiang Cai ${ }^{3}$, Guifang Xu1 ${ }^{1}$, Xiaoping Zou ${ }^{1}$, \\ Lei Wang'
}

Institutions

1 Department of Gastroenterology, The Affiliated Drum Tower Hospital of Nanjing University, Medical School, Nanjing, China

2 Department of Geriatrics, The Affiliated Drum Tower Hospital of Nanjing University, Medical School, Nanjing, China

3 Digestive Diseases, Emory University, Atlanta, GA, USA

submitted 6.11.2017

accepted after revision 14.3.2018

\author{
Bibliography \\ DOI https://doi.org/10.1055/a-0619-4803 | \\ Endoscopy International Open 2018; 06: E950-E956 \\ (c) Georg Thieme Verlag KG Stuttgart · New York \\ ISSN 2364-3722
}

Corresponding author

Lei Wang, Department of Gastroenterology, The Affiliated

Drum Tower Hospital of Nanjing University, Medical School, No. 321, Zhongshan Road, Nanjing, Jiangsu 210008, China Fax: +86-138-51579216

867152094@qq.com

\section{ABSTRACT}

Background and study aims Gastrointestinal stromal tumors (GISTs) are the most common mesenchymal tumors in the gastrointestinal tract. Up to the present time, complete surgical excision has been the standard treatment for primary GISTs greater than $2 \mathrm{~cm}$. It is well known that tumor rupture during surgery is an independent risk factor for peritoneal metastasis; however, it is not known whether the risk of peritoneal metastasis increases in cases where the tumor is ruptured during endoscopic resection.

Patients and methods A total of 195 patients treated for GIST between January 2014 and December 2016 in our hospital were enrolled in this study. They were divided into two groups according to whether the tumor was ruptured during endoscopic resection. The rate of peritoneal metastasis in patients in the two groups who also suffered perforation was investigated from the follow-up results.

Results Approximately $55.4 \%$ of all patients were female and the average age of the study group was $59.0 \pm 10.3$ years. Of the 195 patients, the tumors in 27 were ruptured and the remaining 168 patients underwent en bloc resection. There was no statistically significant difference in gender or age between the two groups. The median tumor size (maximum diameter) in all patients was $1.5 \mathrm{~cm}(0.3-$ $5.0 \mathrm{~cm}): 2.5 \mathrm{~cm}(0.8-5.0 \mathrm{~cm})$ and $1.4 \mathrm{~cm}(0.3-4.0 \mathrm{~cm})$ in the tumor rupture group and en bloc resection group, respectively $(P<0.001)$. Most of the tumors were located in the gastric fundus. At a median follow-up of $18.7 \pm 10.2$ months, neither tumor recurrence (liver metastasis, peritoneal metastasis, local recurrence) nor mortality related to GISTs were detected.

Conclusions Tumor rupture during endoscopic resection of gastric GISTs may not be a risk factor for peritoneal metastasis.

\section{Introduction}

Gastrointestinal stromal tumors (GISTs) are the most common mesenchymal tumors in the gastrointestinal tract [1]. Diagnosis of GISTs relies on histopathological and immunohistochemical examination [2], detecting the expression of CD117 and CD34 $[3,4]$.

Since GISTs are not sensitive to traditional radiotherapy and chemotherapy $[5,6]$, surgical resection remains the first choice for treatment of primary GISTs [7]. In recent years, minimally invasive treatments, including both endoscopic and laparoscopic resection, have been widely applied in the treatment of GISTs [8-10].

According to previous studies, tumor rupture during resection has been considered to be an independent risk factor predicting a high rate of peritoneal metastasis after surgery [11, 12]; however, it is not known whether tumor rupture during endoscopic resection leads to a high risk of peritoneal metasta- 


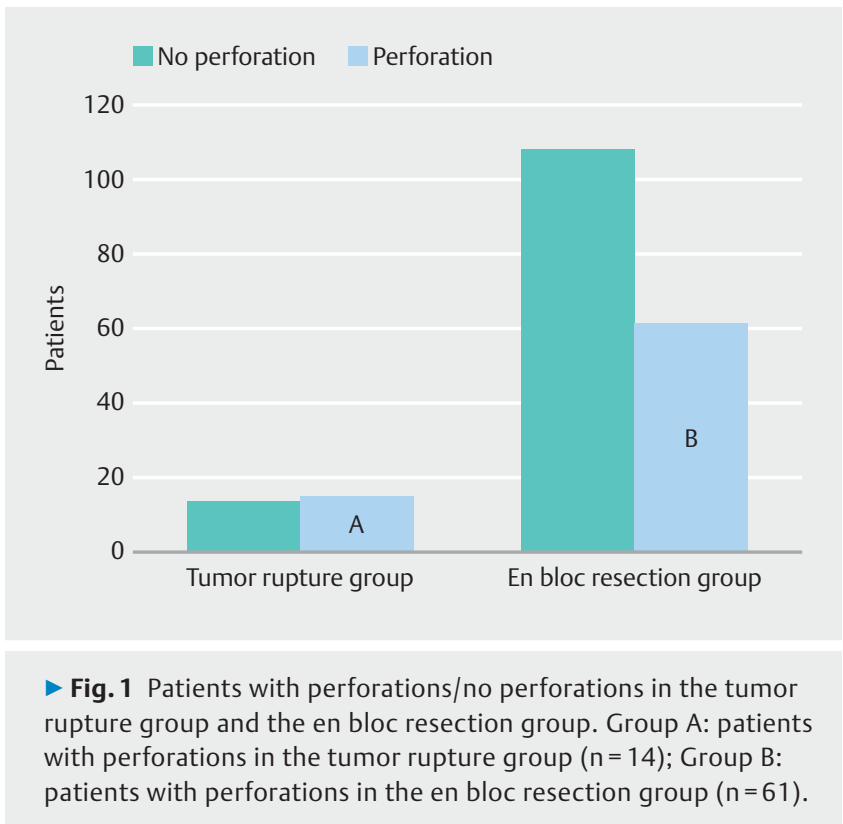

sis. Therefore, we performed a single-center retrospective study to clarify the rate of peritoneal metastasis of gastric GISTs ruptured during endoscopic resection.

\section{Materials and methods}

In total, 195 consecutive patients with primary gastric stromal tumors treated with endoscopic resection in our hospital between January 2014 and December 2016 were enrolled in this study. The diagnosis of all cases was confirmed by immunohistochemical staining. According to the integrity of the tumors, the patients were divided into two groups. Patients with perforations in the tumor rupture group were included in group $A$, whereas patients with perforations in the en bloc resection group formed group B ( $\triangleright$ Fig. 1). The rate of peritoneal metastasis in groups $A$ and $B$ was compared from the follow-up results.

Tumor size was recorded as the maximum diameter. For the en bloc resection group, the maximum diameter was determined based on the macropathology. For the tumor rupture group, the maximum diameter was confirmed according to the preoperative abdominal computed tomography (CT) or endoscopic ultrasound (EUS). Three patients receiving en bloc resection had double lesions, and the tumor diameter data was determined for the larger lesion. Informed consent was obtained from all patients included in this study. The study was approved by the Ethics Committee of Nanjing Drum Tower Hospital.

\section{Follow-up}

This was performed through either chart review or telephone interview on 1 March 2017. All patients received an abdominal CT scan and/or endoscopy every 3 months in the first year, then every 6 months for the tumor rupture group and every 12 months for the en bloc resection group. The end points of this study were patient death and occurrence of peritoneal metastasis. Only deaths related to GISTs were counted as events.

\section{Statistical analyses}

For continuous variables, if the variables followed a Gaussian distribution, the two-sample $t$ test was selected; otherwise, the nonparametric test was chosen. For categorical variables, the Chi-squared test or Fisher's exact test was applied to identify the different characteristics between the groups. $P<0.05$ was considered to represent a statistically significant difference between groups. All statistical analyses were completed using SPSS version 22.0 software (IBM Corp, Armonk, NY, United States).

\section{Management of endoscopic resection}

Preoperative: Preoperative EUS was absolutely necessary. If the tumor was strongly indicative of GIST and was considered to be resectable, the preoperative biopsy could be omitted according to the European Society of Gastrointestinal Endoscopy (ESGE) guidelines [13]. The GISTs of almost all patients originates from the muscularis propria layer (MP). According to the relationship between tumor location and MP, the tumor can be classified into four types. Type $A$ is a tumor with a very narrow connection with the MP layer and which protrudes into the luminal side. Type B has a wider connection with the MP and protrudes into the luminal side at an obtuse angle. Type $C$ is located in the middle of the wall of the gastrointestinal tract. Type $D$ protrudes mainly into the serosal side of the gastrointestinal wall [14]. In our center, endoscopic resection was mainly chosen for patients with tumors of type A (25.2\%), B (48.3\%), and $C(24.4 \%)$, and no more than $4 \mathrm{~cm}$ in diameter, having clear boundaries with adjacent tissues and organs.

Intraoperative: Currently, the typical methods for subepithelial tumors (SETs) include endoscopic submucosal dissection (ESD), endoscopic submucosal excavation (ESE), endoscopic full thickness resection (EFR), and endoscopic submucosal tunneling dissection (ESTD) [15-17]. The operations were performed by our experienced endoscopic specialists. The technique most applied in our center was $\operatorname{ESD}(122,20 / 102$ in the rupture group/en bloc resection group), followed by EFR (63, $7 / 56$ in the rupture group/en bloc resection group), and the least used was ESE ( 4 in the en bloc resection group). ESTD was performed on the remaining six patients.

During the operation, if a pneumoperitoneum developed, a 20-gauge needle was inserted into the right lower quadrant to release gas.

Usually, the retrieval of tumors larger than $2 \mathrm{~cm}$ has a high risk of inducing severe damage to the cardia or the upper esophageal sphincter. In clinical practice, if broken tumors were difficult to extract, the tumors would be cut further in the stomach cavity before being removed. We tried to retain the integrity of the en bloc samples for more accurate pathological evaluation; however, four tumors $(2.5 / 2.6 / 2.8 / 3.0 \mathrm{~cm}$ in transverse diameter) in the en bloc resection group were cut in half in the stomach cavity because they were difficult to remove directly. 
- Table 1 Characteristics of 195 patients with primary resectable gastric GIST [1].

\begin{tabular}{|c|c|c|c|c|}
\hline Factor & $\begin{array}{l}\text { All patients } \\
(n=195)\end{array}$ & $\begin{array}{l}\text { Tumor rupture group } \\
(\mathrm{n}=27)\end{array}$ & $\begin{array}{l}\text { En bloc resection group } \\
(n=168)\end{array}$ & $P$ value \\
\hline Age, mean $\pm S D$, years & $59.0 \pm 10.3$ & $59.1 \pm 12.6$ & $58.9 \pm 9.9$ & 0.918 \\
\hline Gender & & & & 0.218 \\
\hline - Male & $87(44.6 \%)$ & $15(55.6 \%)$ & $72(42.9 \%)$ & \\
\hline - Female & $108(55.4 \%)$ & $12(44.4 \%)$ & $96(57.1 \%)$ & \\
\hline Tumor size, median (range), cm & $1.5(0.3-5.0)$ & $2.5(0.8-5.0)$ & $1.4(0.3-4.0)$ & $<0.001$ \\
\hline Tumor location & & & & 0.827 \\
\hline - Gastric fundus & $116(59.5 \%)$ & $16(59.3 \%)$ & $100(59.5 \%)$ & \\
\hline - Gastric body & $42(21.5 \%)$ & $6(22.2 \%)$ & $36(21.4 \%)$ & \\
\hline - Gastric antrum & $14(7.2 \%)$ & $3(11.1 \%)$ & $11(6.5 \%)$ & \\
\hline - Gastric cardia & $21(10.8 \%)$ & $2(7.4 \%)$ & $19(11.3 \%)$ & \\
\hline - Gastric angle & $2(1.0 \%)$ & $0(0.0 \%)$ & $2(1.2 \%)$ & \\
\hline Perforation & & & & 0.123 \\
\hline - Yes & $75(38.5 \%)$ & $14(51.9 \%)$ & $61(36.3 \%)$ & \\
\hline - No & $120(61.5 \%)$ & $13(48.1 \%)$ & $107(63.7 \%)$ & \\
\hline Follow-up time, mean $\pm S D$, months & $18.7 \pm 10.2$ & $19.7 \pm 10.2$ & $18.6 \pm 10.1$ & 0.619 \\
\hline Recurrence & $0(0.0 \%)$ & $0(0.0 \%)$ & $0(0.0 \%)$ & 1.000 \\
\hline
\end{tabular}

Postoperative: Patients were asked to fast for 24 hours. Antibiotics and proton pump inhibitors were used routinely in patients with perforation/GWD (gastric wall defect) to prevent infection and hemorrhage. For patients who had a GWD, a gastrointestinal decompression drainage tube was placed followed by fasting for several days.

Careful preoperative, intraoperative, and postoperative management can significantly reduce the complications of abdominal pain, bleeding, and infection. So, only a few patients showed signs of abdominal complications (abdominal pain, abdominal distension) and few suffered sepsis.

\section{Results}

\section{Patients}

In this study, 197 patients were screened, and two of them were excluded because of early gastric cancer and residual stomach $(1.2 \mathrm{~cm}, 0.8 \mathrm{~cm}$ in diameter at the gastric fundus and gastric body, respectively). So, 195 patients were enrolled and their data analyzed. In total, 27 patients were placed in the tumor rupture group, among whom 14 had perforations (group $A, N_{A}=14$ ). The remaining 168 patients were placed in the en bloc resection group, of whom 61 had perforations (group $B$, $\left.N_{B}=61\right)$. The percentage of male patients in the tumor rupture group (55.6\%) was higher than in the en bloc resection group (42.9\%), however, there was no statistically significant difference between the two groups $(P=0.218)$. The mean ages of the tumor rupture group and the en bloc resection group were
$59.1 \pm 12.6$ years and $58.9 \pm 9.9$ years, respectively, and no statistically significant difference was present $(P=0.918)$. The clinical and pathological features of all cases are shown in - Table 1, and the age and gender distributions are shown in - Fig. 2.

\section{Tumors}

The median tumor size (maximum diameter) for all patients was $1.5 \mathrm{~cm}(0.3-5.0 \mathrm{~cm}): 2.5 \mathrm{~cm}(0.8-5.0 \mathrm{~cm})$ and $1.4 \mathrm{~cm}$ $(0.3-4.0 \mathrm{~cm})$ for the tumor rupture group and en bloc resection group, respectively. The tumor size in the tumor rupture group was significantly larger than that in the en bloc resection group $(P<0.001)$. All patients had a tumor diameter no more than $4 \mathrm{~cm}$ apart from one patient in the tumor rupture group with a $5 \mathrm{~cm}$ tumor. The scatterplot of maximum tumor diameter is shown in > Fig. 3 .

Tumors were predominantly located at the gastric fundus (116 cases, $59.5 \%$ of all cases), followed by the gastric body (42 cases, $21.5 \%$ of all cases), the gastric antrum (14 cases, $7.2 \%$ of all cases), the gastric cardia ( 21 cases, $10.8 \%$ of all cases), and rarely in the gastric angle ( 2 cases, $1.0 \%$ of all cases). There was no statistically significant difference between the two groups related to distribution of tumor location ( $\triangleright \mathrm{Ta}$ ble $1, P=0.827)$.

Immunohistochemical staining is essential for diagnosis of GISTs [18]. The researchers reviewed the pathology reports and the data are summarized as follows. First, six patients, accounting for $3.1 \%(6 / 195)$ of all cases, were CD117 negative 


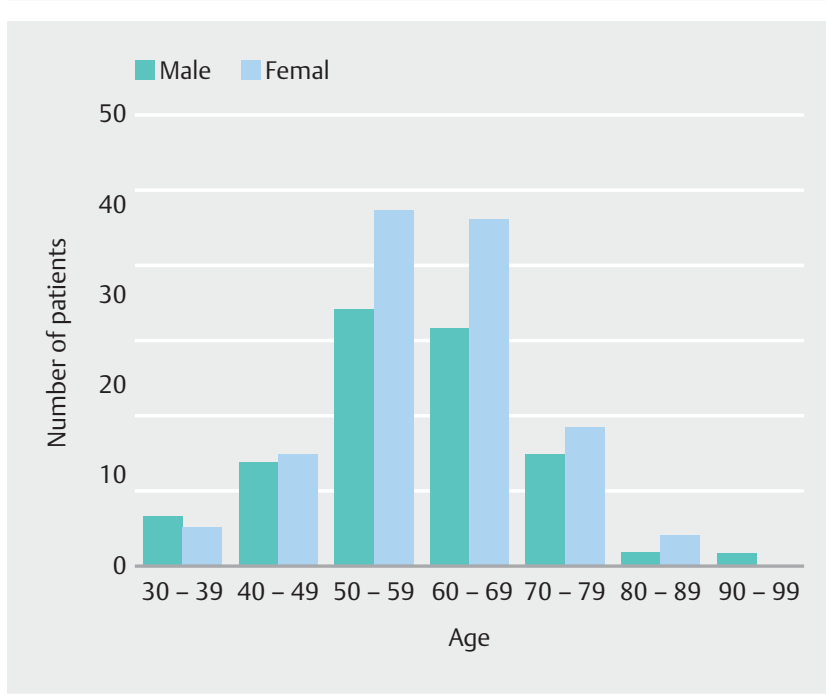

- Fig. 2 Age and gender distribution of all patients in the study $(n=195)$.

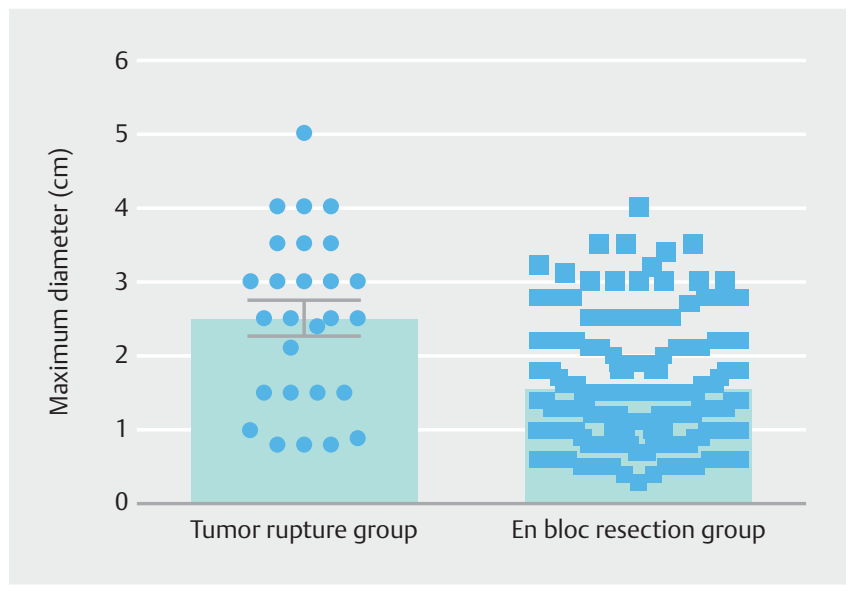

- Fig. 3 Scatterplot of maximum tumor diameter in the two groups.

which was similar to previous research [19]. Second, only two patients had negative DOG1 (Discovered On GIST) accounting for $1.0 \%(2 / 195)$ of all cases. Third, there was only one patient having a CD34 negative tumor. More details are shown in $>$ Table 2.

Only $4(2.1 \%, 4 / 195)$ of the patients had a high mitotic index (>10/50 high power fields (HPF)), and $92.8 \%(181 / 195)$ of all patients had a mitotic index no more than 5 .

There were only two positive margins in the en bloc resection group. The ruptured tumors including four tumors in the en bloc resection group, which were cut in half in the stomach cavity because they were difficult to remove directly, and tumors in the tumor rupture group were not evaluated by the cutting edge.
- Table 2 Immunohistochemistry of the tumors.

\begin{tabular}{|l|l|l|l|l|}
\hline & $\begin{array}{l}\text { Tumor } \\
\text { rupture } \\
\text { group }\end{array}$ & $\begin{array}{l}\text { En bloc } \\
\text { resection } \\
\text { group }\end{array}$ & P value \\
\hline \multirow{2}{*}{ CD117 } & Yes $(n=189)$ & 26 & 163 & 0.596 \\
\hline \multirow{2}{*}{ DOG1 } & No $(n=6)$ & 1 & 5 & \\
\hline & Yes $(n=194)$ & 26 & 167 & 0.258 \\
\hline \multirow{2}{*}{ CD34 } & No $(n=1)$ & 1 & 1 & \\
\hline & Yes $(n=193)$ & 26 & 168 & 0.138 \\
\hline & No $(n=2)$ & 1 & 0 & \\
\hline
\end{tabular}

CD117: CD117 protein; DOG1: Discovered On GIST; CD34: CD34 protein.

\section{Follow-up and recurrence}

The follow-up and recurrence outcomes are summarized in - Table 1. All 195 patients had follow-up data. The mean follow-up time for the tumor rupture group was $19.7 \pm 10.2$ months, and $18.6 \pm 10.1$ months for the en bloc resection group. During the follow-up interval, no tumor recurrence (liver metastasis, peritoneal metastasis, local recurrence) and no mortality related to GISTs were reported in the two groups. For all patients in groups $A$ and $B$, the mean follow-up time was $17.9 \pm 10.3$. There was no statistically significant difference concerning peritoneal metastasis rate between group $A$ and $B$ ( $\triangleright$ Table 3 ).

\section{Discussion}

Based on epidemiological studies, the incidence of GISTs is estimated to be approximately 20 cases/1000000 inhabitants/ year, with no obvious gender differences $[5,20]$. However, our data showed a female predilection, which is consistent with the findings from studies in other Asian countries [21,22], possibly due to race and geography.

In this study, the fundus of stomach is the most common location of GISTs. In total, 96.9\% (189/195) and 99.5\% (194/195) of the included population were positive for CD117 and CD34, respectively, consistent with a previous report of a multicenter study performed in China [23].

In our center, endoscopic resection was mainly chosen for patients with tumors no more than $4 \mathrm{~cm}$ in diameter, apart from one patient with a lesion of $5 \mathrm{~cm}$ (originating from the MP, type A). This was a 91-year-old man who would not tolerate surgical resection. Eventually, the patient underwent endoscopic resection in September 2016. The tumor was broken during resection and had no perforations; the postoperative pathology report showed that the mitotic count was about 1/50 HPF indicating a low metastatic rate according to Mietinnen and Lasota [1]. Until the last follow-up, no evidence of recurrence was found with abdominal $C T$, and we shall continue to monitor this patient carefully.

Tumor rupture has been considered to be an independent risk factor predicting a high risk of peritoneal metastasis after 
- Table 3 Characteristics and follow-up outcomes of groups A and B.

\begin{tabular}{|c|c|c|c|c|}
\hline Factor & Total $(n=75)$ & Group $A^{1}(n=14)$ & Group $B^{1}(n=61)$ & $P$ value \\
\hline Age, mean $\pm S D$, years & $58.0 \pm 10.0$ & $55.3 \pm 11.2$ & $58.6 \pm 9.7$ & 0.263 \\
\hline \multicolumn{5}{|l|}{ Gender } \\
\hline - Male & 34 & 7 & 27 & 0.697 \\
\hline - Female & 41 & 7 & 34 & \\
\hline Tumor size, median (range), cm & $1.5(0.3-4.0)$ & $3.0(0.8-4.0)$ & $1.5(0.3-3.5)$ & $<0.001$ \\
\hline Follow-up time, mean $\pm S D$, months & $17.9 \pm 10.3$ & $19.0 \pm 9.9$ & $17.6 \pm 10.4$ & 0.655 \\
\hline Peritoneal metastasis & $0(0.0 \%)$ & $0(0.0 \%)$ & $0(0.0 \%)$ & 1.000 \\
\hline
\end{tabular}

- Table 4 Prognostication of gastric GISTs by tumor size and mitotic rate prior to imatinib treatment [1]

\begin{tabular}{|c|c|c|c|c|}
\hline \multicolumn{2}{|c|}{ Tumor parameters } & \multirow{2}{*}{$\begin{array}{l}\text { Percentage of patients } \\
\text { with progressive disease } \\
\text { of gastric GISTs }\end{array}$} & \multicolumn{2}{|c|}{ Number of patients in this study } \\
\hline Tumor size $(\mathrm{cm})$ & $\begin{array}{l}\text { Mitotic rate } \\
\text { (/50 HPF) }\end{array}$ & & Tumor rupture group & En bloc resection group \\
\hline$\leq 2$ & $\leq 5$ & 0 & 9 & 130 \\
\hline$>2$ and $\leq 5$ & $\leq 5$ & 1.9 & 13 & 29 \\
\hline$>5$ and $\leq 10$ & $\leq 5$ & 3.6 & - & - \\
\hline$>10$ & $\leq 5$ & 12 & - & - \\
\hline$\leq 2$ & $>5$ & $0^{1}$ & $1^{2}$ & $6\left(1^{3}+5\right)$ \\
\hline$>2$ and $\leq 5$ & $>5$ & 16 & $4\left(1^{4}+3\right)$ & $3\left(1^{5}+2\right)$ \\
\hline$>5$ and $\leq 10$ & $>5$ & 55 & - & - \\
\hline$>10$ & $>5$ & 86 & - & - \\
\hline \multicolumn{5}{|c|}{$\begin{array}{l}\text { HPF, high power field. } \\
{ }^{1} \text { Small number of cases. } \\
{ }^{2} \text { Tumor size of } 2.0 \mathrm{~cm} \text {, mitotic rate of } 12 / 50 \mathrm{HPF} \text {. } \\
{ }^{3} \text { Tumor size of } 1.5 \mathrm{~cm} \text {, mitotic rate of } 10 / 50 \mathrm{HPF} \text {. } \\
{ }^{4} \text { Tumor size of } 3.5 \mathrm{~cm} \text {, mitotic rate of } 10 / 50 \mathrm{HPF} \text {. } \\
{ }^{5} \text { Tumor size of } 2.2 \mathrm{~cm} \text {, mitotic rate of } 14 / 50 \mathrm{HPF} \text {. }\end{array}$} \\
\hline
\end{tabular}

surgical resection [11, 24]. Hohenberger et al. [25] showed that patients with a GIST which had ruptured into the abdominal cavity had a risk of recurrence of nearly $94 \%$ (15/16, $93.7 \%)$ without adjuvant treatment, and the overall risk of peritoneal metastasis was 65.2\% (15/23; peritoneum, 9; peritoneum and liver, 6). However, to our knowledge, there is no published study discussing the metastasis and recurrence rate of ruptured GISTs resected by endoscopic resection.

As shown by several studies, the median time to recurrence is approximately 2 years and recurrence usually occurs in either the peritoneal surface or/and the liver [26,27]. In our study, with a mean follow-up of $17.9 \pm 10.3$ months, no statistically significant difference in the rate of peritoneal metastasis was found between groups A and B, which is at odds with observations in surgical resection. One possible reason might be the tumor size. The tumors in open resections are obviously larger than those in endoscopic resections. The other possibility could be that the tumor was broken or ruptured inside the stomach wall during the endoscopic resection before the perforation occurred.

All GISTs are thought to be potential malignant lesions, and the modified NIH risk stratification system has been widely used to assess the risk associated with GISTs. Studies have identified that patients with high risks (including ruptured tumors) of metastasis or recurrence had substantially poorer outcome after surgery than those with intermediate risk or low/very low risk [28-30]. According to the modified NIH Risk Classification system, we found that there was no difference concerning the peritoneal metastasis rate and overall survival rate between patients with ruptured tumors (high risk) and those with en bloc resection tumors (very low risk or low risk) in our study ( $\triangleright$ Table 3). Our data also demonstrated a favorable outcome for the four patients with a high mitotic index (high risk). Thus, we hypothesize that, for gastric GISTs receiving endoscopic treatment (always having a relatively smaller tumor diameter), the modified NIH Risk Classification system may not be suitable for 
assessing the prognosis. Perhaps the estimation of tumor size to some degree leads to the optimal prognostic prediction of gastric GISTs, independently of tumor rupture and mitotic index. Namely, in the cases of smaller tumors, the prediction value of tumor rupture and mitotic index diminishes. Nevertheless, due to the limited sample size, we could not draw a definite conclusion, and further efforts are needed to clarify this issue.

When the risk of gastric GIST is assessed, we recommend that tumors should be classified firstly according to the WHO classification of tumors of the digestive system and then individualized treatment should be based on whether the tumor is broken or not. In our data (> Table 4 [1]), 96.4\% (188/195) of patients had less than $1.9 \%$ of patients with progressive disease, so postoperative follow-up was safe. There were four patients (A, B, C, and D) with a high mitotic index (>10/50 HPF). We recommended patients $A, B$, and $D$ take imatinib after the gene test. Patient A refused due to its high cost. Patients $B$ and $D$ accepted imatinib at an initial dose of $400 \mathrm{mg} /$ day but they both stopped taking the drug due to financial considerations after 6 and 3 months, respectively. During the follow-up, we paid close attention to the changes in their condition and fortunately found no signs of recurrence up to the present time.

Although our study showed favorable outcomes, it still has some limitations such as small sample size and relatively short follow-up time. Although the longest follow-up time was 38.6 months, the shortest was only 2.3 months. Comparison of the two metastasis rates should best be performed using a statistical model. The effort of the two-logistic regression method came to nothing because the outcome variable was only one. This situation may be attributed to the limited sample size or insufficient follow-up time, causing the conclusion to be not very stable. Combining the existing research results, the relatively low degree of malignancy, and the small diameter of gastric stromal tumors in endoscopic resection, we considered that the risk of peritoneal metastasis may not be increased even though the tumors ruptured with perforation. In a future study, we will further expand the sample size and increase the follow-up time to identify the relationship between perforation with rupture and the risk of peritoneal metastasis.

In conclusion, tumor rupture of gastric GISTs during endoscopic resection may not be a risk factor for peritoneal metastasis. The modified NIH risk classification system might not be suitable for assessing the risk of tumors resected by endoscopy (usually with a small diameter). Further efforts are necessary to assess the safety of endoscopic resection.

\section{Competing interests}

None

References

[1] Miettinen M, Lasota J. Gastrointestinal stromal tumors: pathology and prognosis at different sites. Semin Diagn Pathol 2006; 23: 70-83
[2] Chourmouzi D, Sinakos E, Papalavrentios L et al. Gastrointestinal stromal tumors: a pictorial review. J Gastrointest Liver Dis 2009; 18: $379-383$

[3] Miettinen M, Lasota J. Gastrointestinal stromal tumors: review on morphology, molecular pathology, prognosis, and differential diagnosis. Arch Pathol Lab Med 2006; 130: 1466 - 1478

[4] Beham AW, Schaefer IM, Schuler P et al. Gastrointestinal stromal tumors. Int J Colorectal Dis 2012; 27: 689-700

[5] Bamboat ZM, Dematteo RP. Updates on the management of gastrointestinal stromal tumors. Surg Oncol Clin N Am 2012; 21: $301-316$

[6] Karakousis GC, Singer S, Zheng J et al. Laparoscopic versus open gastric resections for primary gastrointestinal stromal tumors (GISTs): a size-matched comparison. Ann Surg Oncol 2011; 18: 1599-1605

[7] Frankel TL, Chang AE, Wong SL. Surgical options for localized and advanced gastrointestinal stromal tumors. J Surg Oncol 2011; 104: $882-887$

[8] Wilhelm D, von Delius S, Burian M et al. Simultaneous use of laparoscopy and endoscopy for minimally invasive resection of gastric subepithelial masses - analysis of 93 interventions. World J Surg 2008; 32: $1021-1028$

[9] Novitsky YW, Kercher KW, Sing RF et al. Long-term outcomes of laparoscopic resection of gastric gastrointestinal stromal tumors. Ann Surg 2006; 243: 738-745; discussion 745-737

[10] Kim IH, Kim IH, Kwak SG et al. Gastrointestinal stromal tumors (GISTs) of the stomach: a multicenter, retrospective study of curatively resected gastric GISTs. Ann Surg Treat Res 2014; 87: 298-303

[11] Joensuu $H$. Risk stratification of patients diagnosed with gastrointestinal stromal tumor. Hum Pathol 2008; 39: 1411 - 1419

[12] Rutkowski P, Nowecki ZI, Michej W et al. Risk criteria and prognostic factors for predicting recurrences after resection of primary gastrointestinal stromal tumor. Ann Surg Oncol 2007; 14: 2018-2027

[13] Dumonceau JM, Polkowski M, Larghi A et al. Indications, results, and clinical impact of endoscopic ultrasound (EUS)-guided sampling in gastroenterology: European Society of Gastrointestinal Endoscopy (ESGE) Clinical Guideline. Endoscopy 2011; 43: 897-912

[14] Kim HH. Endoscopic treatment for gastrointestinal stromal tumor: Advantages and hurdles. World J Gastrointest Endosc 2015; 7: 192 205

[15] Meng FS, Zhang ZH, Hong YY et al. Comparison of endoscopic submucosal dissection and surgery for the treatment of gastric submucosal tumors originating from the muscularis propria layer: a singlecenter study (with video). Surg Endosc 2016; 30: 5099-5107

[16] Schmidt A, Meier B, Caca K. Endoscopic full-thickness resection: Current status. World J Gastroenterol 2015; 21: 9273 - 9285

[17] Ko WJ, Cho JY. Current techniques for treating gastrointestinal stromal tumors in the upper gastrointestinal tract. Clin Endosc 2016; 49: $226-228$

[18] Koo DH, Ryu MH, Kim KM et al. Asian consensus guidelines for the diagnosis and management of gastrointestinal stromal tumor. Cancer Res Treat 2016; 48: 1155 - 1166

[19] Kang GH, Srivastava A, Kim YE et al. DOG1 and PKC-theta are useful in the diagnosis of KIT-negative gastrointestinal stromal tumors. Mod Pathol 2011; 24: $866-875$

[20] Cichoz-Lach H, Kasztelan-Szczerbinska B, Slomka M. Gastrointestinal stromal tumors: epidemiology, clinical picture, diagnosis, prognosis and treatment. Pol Arch Med Wewn 2008; 118: 216-221

[21] Cho MY, Sohn JH, Kim JM et al. Current trends in the epidemiological and pathological characteristics of gastrointestinal stromal tumors in Korea, 2003-2004. J Korean Med Sci 2010; 25: 853-862

[22] Kim KH, Kim MC, Jung G] et al. Long term survival results for gastric GIST: is laparoscopic surgery for large gastric GIST feasible? World ] Surg Oncol 2012; 10: 230 
[23] Dai W], Liu G, Wang M et al. Endoscopic versus laparoscopic resection of gastric gastrointestinal stromal tumors: a multicenter study. Oncotarget 2017; 8: $11259-11267$

[24] Takahashi T, Nakajima K, Nishitani A et al. An enhanced risk-group stratification system for more practical prognostication of clinically malignant gastrointestinal stromal tumors. Int J Clin Oncol 2007; 12: $369-374$

[25] Hohenberger P, Ronellenfitsch U, Oladeji O et al. Pattern of recurrence in patients with ruptured primary gastrointestinal stromal tumour. Br J Surg 2010; 97: 1854-1859

[26] Ng EH, Pollock RE, Munsell MF et al. Prognostic factors influencing survival in gastrointestinal leiomyosarcomas. Implications for surgical management and staging. Ann Surg 1992; 215: 68-77
[27] DeMatteo RP, Lewis J], Leung D et al. Two hundred gastrointestinal stromal tumors: recurrence patterns and prognostic factors for survival. Ann Surg 2000; 231: $51-58$

[28] Hassan I, You YN, Shyyan R et al. Surgically managed gastrointestinal stromal tumors: a comparative and prognostic analysis. Ann Surg Oncol 2008; 15: $52-59$

[29] Huang HY, Li CF, Huang WW et al. A modification of NIH consensus criteria to better distinguish the highly lethal subset of primary localized gastrointestinal stromal tumors: a subdivision of the original high-risk group on the basis of outcome. Surgery 2007; 141: 748 756

[30] Mucciarini C, Rossi G, Bertolini F et al. Incidence and clinicopathologic features of gastrointestinal stromal tumors. A population-based study. BMC Cancer 2007; 7: 230 